\title{
ON THE GROTHENDIECK RING OF VARIETIES
}

\author{
AMIT KUBER 1 , \\ School of Mathematics, University of Manchester, \\ Manchester M13 9PL, England.
}

\begin{abstract}
Let $\mathrm{K}_{0}\left(\operatorname{Var}_{k}\right)$ denote the Grothendieck ring of $k$-varieties over an algebraically closed field $k$. Larsen and Lunts asked if two $k$-varieties having the same class in $\mathrm{K}_{0}\left(\operatorname{Var}_{k}\right)$ are piecewise isomorphic. Gromov asked if a birational self-map of a $k$-variety can be extended to a piecewise automorphism. We show that these two questions are equivalent over any algebraically closed field. If these two questions admit a positive answer, then we prove that its underlying abelian group is a free abelian group. Furthermore, if $\mathfrak{B}$ denotes the multiplicative monoid of birational equivalence classes of irreducible $k$-varieties then we also prove that the associated graded ring of the Grothendieck ring is the monoid ring $\mathbb{Z}[\mathfrak{B}]$.
\end{abstract}

\section{INTRODUCTION}

Let $k$ be a field. A variety over $k$ is a reduced separated scheme of finite type. A subvariety of a variety $X$ is said to be locally closed if it can be written as the intersection of an open subvariety with a closed subvariety. Let $\operatorname{Var}_{k}$ denote the category of $k$-varieties. The Grothendieck group $\mathrm{K}_{0}^{+}\left(\operatorname{Var}_{k}\right)$ is the quotient of the free abelian group generated by the isomorphism classes of $k$-varieties by the following relations.

$$
[X]-[Y]=[X \backslash Y] \text { whenever } Y \subseteq X \text { is a closed subvariety. }
$$

It can be given a ring structure by taking the reduced product $\left(X \times_{\text {Spec } k} Y\right)_{\text {red }}$ of varieties. Recall that if the field $k$ is algebraically closed, then we can simply talk about the product $X \times_{\operatorname{Spec} k} Y$. We denote the Grothendieck ring of varieties by $\mathrm{K}_{0}\left(\operatorname{Var}_{k}\right)$.

Recall that if $R$ is a commutative ring, then an $R$-valued motivic measure is a ring homomorphism $\mathrm{K}_{0}\left(\operatorname{Var}_{k}\right) \rightarrow R$. The Grothendieck ring plays an important role in the theory of motivic integration being the value ring of the universal motivic measure on $k$-varieties. But very little is known about this ring. Poonen [8, Theorem 1] and Kollár [3, Example 6] show that this ring is not a domain when $k$ has characteristic 0 .

Characterizing equality in the Grothendieck ring is an important issue. We need some notation to state this problem precisely.

Set $[n]=\{1,2, \ldots, n\}$ for each $n \in \mathbb{Z}_{>0}$. Two varieties $X$ and $Y$ are said to be piecewise isomorphic, written $X \doteqdot Y$, if there are partitions $X=\bigsqcup_{i \in[n]} X_{i}$ and $Y=\bigsqcup_{j \in[n]} Y_{j}$ of $X$ and $Y$ into locally closed subvarieties such that there is a permutation $\sigma$ of $[n]$ with $X_{i}$ isomorphic to $Y_{\sigma(i)}$ as a variety.

If $X \doteqdot Y$, then clearly $[X]=[Y]$ in $\mathrm{K}_{0}\left(\operatorname{Var}_{k}\right)$. Larsen and Lunts asked whether the converse is true.

2010 Mathematics Subject Classification. 14F42, 14E05, 16Y60, 20M25.

Key words and phrases. Grothendieck ring; varieties; birational geometry; monoid ring.

${ }^{1}$ Email amit.kuber@postgrad.manchester.ac.uk. Research partially supported by a School of Mathematics, University of Manchester Scholarship. 
Question 1.1. 6, Question 1.2] Suppose $X$ and $Y$ are two $k$-varieties such that $[X]=[Y]$ in $\mathrm{K}_{0}\left(\operatorname{Var}_{k}\right)$. Is it true that $X \doteqdot Y$ ?

In the case when $k$ is algebraically closed, we reformulate this question as the cancellative property of the Grothendieck semiring $\mathcal{S}_{k}$ of piecewise isomorphic classes of $k$-varieties in Question 2.2.

Liu and Sebag answered this question over an algebraically closed field of characteristic 0 for varieties with dimension at most one ([7, Propositions 5,6]) and for some classes of dimension two varieties ([7, Theorems 4,5]). Sebag extended this result further ([10, Theorem 3.3]).

This question is quite natural and has many important applications to birational geometry. Consider the following question asked by Gromov as an example.

Question 1.2. [2, $\left.\S 3 . \mathrm{G}^{\prime \prime \prime}\right]$ Let $X$ and $Y$ be algebraic varieties which admit an embedding into a third one, say $X \hookrightarrow Z$ and $Y \hookrightarrow Z$, such that the complements $Z \backslash X$ and $Z \backslash Y$ are biregularly isomorphic. How far are $X$ and $Y$ from being birationally equivalent? Under what conditions are $X$ and $Y$ piecewise isomorphic?

Lamy and Sebag [5] studied the following conjectural reformulation of this question in characteristic 0 .

Conjecture 1.3 (see [5, Conjecture 1]). Let $k$ be an algebraically closed field and let $X$ be a $k$-variety. Let $\phi: X \rightarrow X$ be a birational map. Then it is possible to extend the map $\phi$ to a piecewise automorphism of $X$.

It is known (see [5]) that a positive answer to Question 1.1 will settle this conjecture in the affirmative. We prove the converse in Theorem 3.4 showing that the two statements are in fact equivalent. This may be known to the experts, but we could not find a proof in the literature.

Larsen and Lunts [6] obtained an important motivic measure described in the following theorem.

Theorem 1.4 ([6, Theorem 2.3]). Suppose $k$ is an algebraically closed field of characteristic 0 . Let $\mathfrak{s b}$ denote the multiplicative monoid of stable birational equivalence classes of irreducible varieties. There exists a unique surjective ring homomorphism $\Psi: \mathrm{K}_{0}\left(\operatorname{Var}_{k}\right) \rightarrow \mathbb{Z}[\mathfrak{s b}]$ that assigns to the class in $\mathrm{K}_{0}\left(\operatorname{Var}_{k}\right)$ of a smooth irreducible proper variety its stable birational equivalence class in $\mathbb{Z}[\mathfrak{s} \mathfrak{b}]$.

Bittner [1] obtained the following presentations of the Grothendieck group. Larsen and Lunts mention that Bittner's presentation subsumes the theorem above 6 , Remark 2.4] and this assertion has been proved in detail by Sahasrabudhe in 9 .

Theorem 1.5 ([1, Theorem 3.1]). Let $k$ be a field of characteristic 0 . The Grothendieck group $\mathrm{K}_{0}^{+}\left(\operatorname{Var}_{k}\right)$ has the following presentations:

$(\mathrm{sm})$ as the abelian group generated by the isomorphism classes of smooth varieties over $k$ subject to the relations $[X]=[Y]+[X \backslash Y]$, where $X$ is smooth and $Y \subseteq X$ is a smooth closed subvariety;

(bl) as the abelian group generated by the isomorphism classes of smooth complete $k$-varieties subject to the relations $[\emptyset]=0$ and $\left[\mathrm{Bl}_{Y} X\right]-[E]=$ $[X]-[Y]$, where $X$ is smooth and complete, $Y \subseteq X$ is a smooth closed subvariety, $\mathrm{Bl}_{Y} X$ is the blow-up of $X$ along $Y$ and $E$ is the exceptional divisor of this blow-up.

In Theorem 4.1. we show that if Question 1.1 admits a positive answer over an algebraically closed field $k$ then the Grothendieck group $\mathrm{K}_{0}^{+}\left(\operatorname{Var}_{k}\right)$ is a free abelian group. Further if $k$ has characteristic 0, then this result subsumes Bittner's presentation in view of Hironaka's theorem on resolution of singularities. 
Conventions: In the sequel $k$ denotes an algebraically closed field unless otherwise mentioned. If $X$ is a variety, we use $\operatorname{dim} X$ to denote its dimension and $d(X)$ to denote the number of its irreducible components of maximal dimension.

\section{The SEMIRING OF PIECEWISE ISOMORPHISM CLASSES OF VARIETIES}

The Grothendieck group $\mathrm{K}_{0}(M)$ associated with a commutative monoid $(M,+, 0)$ is an abelian group equipped with a monoid homomorphism $q: M \rightarrow \mathrm{K}_{0}(M)$ that satisfies the following universal property: given an abelian group $N$ and a monoid homomorphism $f: M \rightarrow N$, there is a unique group homomorphism $\bar{f}: \mathrm{K}_{0}(M) \rightarrow N$ such that $f=\bar{f} \circ q$.

A commutative monoid $M$ is said to be cancellative if $a+c=b+c \Rightarrow a=b$ for all $a, b, c \in M$. It is easy to see that $M$ is cancellative if and only if the map $q: M \rightarrow \mathrm{K}_{0}(M)$ is injective.

If $(M,+, \cdot, 0,1)$ is a commutative semiring, then the abelian group $\mathrm{K}_{0}(M)$ can be equipped with a natural commutative ring structure. In this case, we say that $\mathrm{K}_{0}(M)$ is the Grothendieck ring of the semiring $M$.

Let $\{A\}$ denote the piecewise isomorphism class of a variety $A$. The set $\mathcal{S}_{k}$ of piecewise isomorphism classes of $k$-varieties carries a natural semiring structure.

$$
\begin{aligned}
0 & :=\{\emptyset\}, \\
\{A\}+\{B\} & :=\left\{A^{\prime} \sqcup B^{\prime}\right\} \text { where } A^{\prime} \in\{A\}, B^{\prime} \in\{B\}, A^{\prime} \cap B^{\prime}=\emptyset .
\end{aligned}
$$

The product of the classes of varieties is defined by $\{A\} \cdot\{B\}:=\left\{\left(A \times_{\text {Spec } k} B\right)_{\text {red }}\right\}$, where $\{\operatorname{Spec} k\}$ is the multiplicative identity.

A general element of $\mathrm{K}_{0}\left(\mathcal{S}_{k}\right)$ can be written as $\{A\}-\{B\}$ for some varieties $A, B$. Furthermore, $\left\{A_{1}\right\}-\left\{B_{1}\right\}=\left\{A_{2}\right\}-\left\{B_{2}\right\}$ if and only if there is some variety $C$ such that $A_{1}^{\prime} \sqcup B_{2}^{\prime} \sqcup C \doteqdot A_{2}^{\prime} \sqcup B_{1}^{\prime} \sqcup C$ in $\mathcal{S}_{k}$ for some $A_{j}^{\prime} \in\left\{A_{j}\right\}, B_{j}^{\prime} \in\left\{B_{j}\right\}$ for $j=1,2$ such that $A_{1}^{\prime}, B_{2}^{\prime}, C$ and $A_{2}^{\prime}, B_{1}^{\prime}, C$ are families of pairwise disjoint varieties.

On the other hand, a general element of $\mathrm{K}_{0}\left(\operatorname{Var}_{k}\right)$ can be expressed as a finite linear combination $\sum_{i} a_{i}\left[A_{i}\right]-\sum_{j} b_{j}\left[B_{j}\right]$ with $a_{i}, b_{j} \in \mathbb{Z}^{+}$and $A_{i}, B_{j} \in \operatorname{Var}_{k}$. We can choose some $A_{i 1}^{\prime}, A_{i 2}^{\prime}, \ldots, A_{i a_{i}}^{\prime} \in\left[A_{i}\right]$ and $B_{j 1}^{\prime}, B_{j 2}^{\prime}, \ldots, B_{j b_{j}}^{\prime} \in\left[B_{j}\right]$ for each $i, j$ such that every two distinct $A_{i k}^{\prime}$ and $B_{j l}^{\prime}$ are disjoint. Let $A:=\bigsqcup_{i, k} A_{i k}^{\prime}$ and $B:=\bigsqcup_{j, l} B_{j l}^{\prime}$. Then the identities $[A]=\sum_{i} a_{i}\left[A_{i}\right]$ and $[B]=\sum_{j} b_{j}\left[B_{j}\right]$ are clearly true in $\mathrm{K}_{0}\left(\operatorname{Var}_{k}\right)$. Therefore a general element of $\mathrm{K}_{0}\left(\operatorname{Var}_{k}\right)$ can be expressed as $[A]-[B]$ for some varieties $A, B$.

Proposition 2.1. Let $k$ be an algebraically closed field. Then the natural map $\psi: \mathrm{K}_{0}\left(\operatorname{Var}_{k}\right) \rightarrow \mathrm{K}_{0}\left(\mathcal{S}_{k}\right)$ defined by $\psi([A]-[B]):=\{A\}-\{B\}$ is an isomorphism of rings.

Proof. Recall that piecewise isomorphic varieties have the same class in $\mathrm{K}_{0}\left(\operatorname{Var}_{k}\right)$. Note as a consequence of (1) that if $Z_{1}$ and $Z_{2}$ are two disjoint varieties, then $\left[Z_{1}\right]=\left[Z_{2}\right]$ if and only if there is some $W$ disjoint from both $Z_{1}$ and $Z_{2}$ such that $Z_{1} \sqcup W \doteqdot Z_{2} \sqcup W$

Let $A_{1}, B_{1}, A_{2}$ and $B_{2}$ be pairwise disjoint varieties.

Now $\left[A_{1}\right]-\left[B_{1}\right]=\left[A_{2}\right]-\left[B_{2}\right]$ in $\mathrm{K}_{0}\left(\operatorname{Var}_{k}\right)$

$\Longleftrightarrow\left[A_{1}\right]+\left[B_{2}\right]=\left[A_{2}\right]+\left[B_{1}\right]$ in $\mathrm{K}_{0}\left(\operatorname{Var}_{k}\right)$

$\Longleftrightarrow$ there is a variety $C$ disjoint from all $A_{i}$ and $B_{j}$ such that $A_{1} \sqcup B_{2} \sqcup C \doteqdot A_{2} \sqcup B_{1} \sqcup C$

$\Longleftrightarrow\left\{A_{1}\right\}+\left\{B_{2}\right\}=\left\{A_{2}\right\}+\left\{B_{1}\right\}$ in $\mathcal{S}_{k}$

$\Longleftrightarrow\left\{A_{1}\right\}-\left\{B_{1}\right\}=\left\{A_{2}\right\}-\left\{B_{2}\right\}$ in $\mathrm{K}_{0}\left(\mathcal{S}_{k}\right)$.

Thus the map $\psi$ is both well-defined and injective. It is clearly surjective and preserves addition. 
Finally we note that $\psi$ also preserves multiplication. Observe that for any two varieties $X$ and $Y$, we have $[X] \cdot[Y]=\left[X \times_{\text {Speck }} Y\right]$ in $\mathrm{K}_{0}\left(\operatorname{Var}_{k}\right)$ and $\{X\} \cdot\{Y\}=$ $\left\{X \times{ }_{\text {Speck }} Y\right\}$ in $\mathrm{K}_{0}\left(\mathcal{S}_{k}\right)$. Hence $\psi$ preserves multiplication of varieties. Using the distributivity of multiplication over addition completes the proof.

The following question is natural.

Question 2.2. Let $k$ be an algebraically closed field. Is the semiring $\mathcal{S}_{k}$ of piecewise isomorphism classes of $k$-varieties cancellative?

A positive answer to this question is equivalent to injectivity of the natural map $q: \mathcal{S}_{k} \rightarrow \mathrm{K}_{0}\left(\mathcal{S}_{k}\right)$. In view of Proposition 2.1 it is also equivalent to injectivity of the map $\psi^{-1} \circ q: \mathcal{S}_{k} \rightarrow \mathrm{K}_{0}\left(\operatorname{Var}_{k}\right)$. Hence a positive answer to Question 2.2 is equivalent to a positive answer to Question 1.1.

\section{Equivalence of Question 1.1 and Conjecture 1.3}

We note a consequence of equality in the Grothendieck ring from [7]. Scanlon has pointed out another proof using counting function methods from [4].

Proposition 3.1 ([7, Cor. 5]). Let $k$ be an algebraically closed field of characteristic 0 . Let $A$ and $B$ be two varieties with $[A]=[B]$ in $\mathrm{K}_{0}\left(\operatorname{Var}_{k}\right)$. Then $\operatorname{dim} A=\operatorname{dim} B$ and $d(A)=d(B)$.

Given two varieties $V$ and $W$ such that $[V]=[W]$ in $\mathrm{K}_{0}\left(\operatorname{Var}_{k}\right)$, Proposition 2.1 states that there is some variety $Z$ disjoint from $V$ and $W$ such that $V \sqcup Z \doteqdot W \sqcup Z$. Under the hypothesis of Conjecture 1.3, we develop a technique in Proposition 3.2 to remove a dense subset of $Z$ from both $V \sqcup Z$ and $W \sqcup Z$ to leave piecewise isomorphic complements. In fact the following Proposition is a reformulation of Conjecture 1.3.

Proposition 3.2. Suppose Conjecture 1.3 holds for an algebraically closed field $k$.

Let $V, W$ and $Z$ be $k$-varieties such that $Z$ is disjoint from both $V$ and $W$, $\operatorname{dim} V \leq \operatorname{dim} W \leq t=\operatorname{dim} Z$ and $d(Z)=e$. Assume that $d(V)=d(W)$ if $\operatorname{dim} V=\operatorname{dim} W$. Further let $d= \begin{cases}d(V) & \text { if } \operatorname{dim} V=\operatorname{dim} W=t, \\ 0 & \text { otherwise. }\end{cases}$

Let $S_{1}, S_{2}, \ldots, S_{d+e}$ and $T_{1}, T_{2}, \ldots, T_{d+e}$ be families of pairwise disjoint irreducible subvarieties of $V \sqcup Z$ and $W \sqcup Z$ respectively such that $\operatorname{dim} S_{l}=\operatorname{dim} T_{l}=t$ for each $l \in[d+e]$. Assume that the varieties $S_{l}$ and $T_{l}$ are either disjoint from or contained in $Z$ for each $l \in[d+e]$. Furthermore assume that $\tau$ is a permutation of $[d+e]$ such that $f_{l}: S_{l} \cong T_{\tau(l)}$ is a variety isomorphism for each $l \in[d+e]$.

Then there are subsets $P, Q \subseteq[d+e]$ of size $e$, a bijection $\lambda: Q \rightarrow P$ and dense subvarieties $S_{l}^{\prime} \subseteq S_{l}, T_{l}^{\prime} \subseteq T_{l}$ for $l \in[d+e]$ such that the following hold:

- $S_{\lambda(l)}^{\prime}=T_{l}^{\prime} \subseteq Z$ for each $l \in Q$;

- $\bigsqcup_{m \notin P} S_{m}^{\prime} \doteqdot \bigsqcup_{l \notin Q} T_{l}^{\prime}$;

- $\bigsqcup_{l \in[d+e]}\left(S_{l} \backslash S_{l}^{\prime}\right) \doteq \bigsqcup_{l \in[d+e]}\left(T_{l} \backslash T_{l}^{\prime}\right)$.

Proof. We have $S_{l} \subseteq Z$ and $T_{m} \subseteq Z$ for exactly $e$ values of both $l$ and $m$. Let $Q, P \subseteq[d+e]$ be the sets of such $m$ and $l$ respectively. For each $l \in P$, there is a unique $m \in Q$ such that $\operatorname{dim}\left(S_{l} \cap T_{m}\right)=t$. Let $\lambda: Q \rightarrow P$ define this correspondence.

Case I: Suppose that $\lambda(l)=\tau^{-1}(l)$ for each $l \in Q$.

In this case we set $S_{\lambda(l)}^{\prime}=T_{l}^{\prime}:=S_{\lambda(l)} \cap T_{l}$ for each $l \in Q$. The isomorphism $f_{\lambda(l)}: S_{\lambda(l)} \rightarrow T_{l}$ can be seen as a birational self-map of $S_{\lambda(l)} \cup T_{l}$. Since Conjecture 1.3 holds, this birational map can be extended to obtain a piecewise automorphism 
of $S_{\lambda(l)} \cup T_{l}$. In particular, one gets a piecewise isomorphism $T_{l} \backslash S_{\lambda(l)} \doteqdot S_{\lambda(l)} \backslash T_{l}$ of lower dimensional subvarieties.

Case II: Suppose that $\lambda(i) \neq \tau^{-1}(i)$ for some $i \in Q$. Fix such $i$ and let $j:=\lambda(i)$.

The idea of the proof is to find subvarieties $S_{l}^{1} \subseteq S_{l}$ and $T_{l}^{1} \subseteq T_{l}$ for each $l \in[d+e]$ and a permutation $\tau_{1}$ of $[d+e]$ such that the following properties are satisfied:

(i) $\left\{l \in Q: \lambda(l)=\tau_{1}^{-1}(l)\right\} \supsetneq\left\{l \in Q: \lambda(l)=\tau^{-1}(l)\right\}$;

(ii) $f_{l}: S_{l} \backslash S_{l}^{1} \cong T_{\tau(l)} \backslash T_{\tau(l)}^{1}$ is an isomorphism for each $l \in[d+e]$;

and then continue inductively.

Since $j \neq \tau^{-1}(i)$, we make the following assignments.

$$
\begin{aligned}
S_{l}^{1}:= \begin{cases}T_{i} \cap S_{j} & \text { if } l=j, \\
f_{\tau^{-1}(i)}^{-1}\left(T_{i} \cap S_{j}\right) & \text { if } l=\tau^{-1}(i) \\
S_{l} & \text { otherwise. }\end{cases} \\
T_{l}^{1}:= \begin{cases}f_{j}\left(T_{i} \cap S_{j}\right) & \text { if } l=\tau(j), \\
T_{i} \cap S_{j} & \text { if } l=i \\
T_{l} & \text { otherwise. }\end{cases}
\end{aligned}
$$

The maps $f_{\tau^{-1}(i)}$ and $f_{j}$ clearly restrict to isomorphisms $S_{\tau^{-1}(i)} \backslash S_{\tau^{-1}(i)}^{1} \cong T_{i} \backslash T_{i}^{1}$ and $S_{j} \backslash S_{j}^{1} \cong T_{\tau(j)} \backslash T_{\tau(j)}^{1}$ of lower dimensional subvarieties. This takes care of property $($ ii $)$.

Now we define $\tau_{1}:[d+e] \rightarrow[d+e]$ as follows.

$$
\tau_{1}(l):= \begin{cases}i & \text { if } l=j, \\ \tau(j) & \text { if } l=\tau^{-1}(i) \\ \tau(l) & \text { otherwise. }\end{cases}
$$

Note that $\lambda(i) \neq \tau^{-1}(i)$, but $\lambda(i)=\tau_{1}^{-1}(i)$. This shows that $(i)$ holds.

Furthermore, $f_{\tau^{-1}(i)}^{1}:=f_{j} \circ f_{\tau^{-1}(i)}: S_{\tau^{-1}(i)}^{1} \rightarrow T_{\tau_{1}\left(\tau^{-1}(i)\right)}^{1}$ and $f_{j}^{1}:=i d: S_{j}^{1} \rightarrow$ $T_{\tau_{1}(j)}^{1}$ are isomorphisms. For the remaining $l \in[d+e]$, we set $f_{l}^{1}:=f_{l}$.

Thus $f_{l}^{1}: S_{l}^{1} \rightarrow T_{\tau_{1}(l)}^{1}$ is an isomorphism for each $l \in[d+e]$. If $\lambda$ does not agree with $\tau_{1}^{-1}$ on $Q$, we iterate the process with varieties $S_{l}^{1}, T_{l}^{1}$, functions $f_{l}^{1}$ and permutation $\tau_{1}$ until some $\left(\tau_{n}\right)^{-1}$ agrees with $\lambda$ on $Q$.

We set $T_{l}^{\prime}:=T_{l}^{n}$ for each $l \notin Q$ and $S_{l}^{\prime}:=S_{l}^{n}$ for each $l \notin P$.

The varieties $S_{\lambda(l)}^{n}, T_{l}^{n}$, for $l \in Q$, together with the function $\lambda=\tau_{n}^{-1} \uparrow_{Q}$ is the set-up for the first case. A construction similar to that case gives the required varieties $S_{\lambda(l)}^{\prime}, T_{l}^{\prime}$.

In both cases, it is clear that the construction guarantees the final two conditions in the statement of the proposition.

Now we derive the result of Proposition 3.1 for an algebraically closed field of non-zero characteristic under the hypothesis of Conjecture 1.3 .

Corollary 3.3. Suppose Conjecture 1.3 holds for an algebraically closed field $k$. Let $V$ and $W$ be two $k$-varieties with $[V]=[W]$ in $\mathrm{K}_{0}\left(\operatorname{Var}_{k}\right)$. Then $\operatorname{dim} V=\operatorname{dim} W$ and $d(V)=d(W)$.

Proof. Without loss we may assume that $V$ and $W$ are disjoint and have the same class in $\mathrm{K}_{0}\left(\operatorname{Var}_{k}\right)$. In view of Proposition 2.1, there is a variety $Z$ disjoint from both $V$ and $W$ such that $V \sqcup Z \doteqdot W \sqcup Z$.

Case I: $\operatorname{dim} Z, \operatorname{dim} V \leq \operatorname{dim} W$.

Counting the number of irreducible components of maximal dimension on both sides of the piecewise isomorphism $V \sqcup Z \doteqdot W \sqcup Z$ forces the equalities $\operatorname{dim} V=$ $\operatorname{dim} W$ and $d(V)=d(W)$. 
Case II: $\operatorname{dim} V \leq \operatorname{dim} W<\operatorname{dim} Z$.

Let $e:=d(Z)$ and $d=0$. The piecewise isomorphism $V \sqcup Z \doteqdot W \sqcup Z$ gives two families $S_{1}, S_{2}, \ldots, S_{e}$ and $T_{1}, T_{2}, \ldots, T_{e}$ of irreducible subvarieties of $Z$, each of dimension $e$, satisfying the hypotheses of Proposition 3.2 .

An application of the Proposition then gives a subvariety $Z_{1}=\bigsqcup_{i \in[e]} T_{i}^{\prime} \subseteq Z$ with $\operatorname{dim} Z_{1}=\operatorname{dim} Z, d\left(Z_{1}\right)=d(Z)$ such that $V \sqcup\left(Z \backslash Z_{1}\right) \doteqdot W \sqcup\left(Z \backslash Z_{1}\right)$. Proposition 3.2 can be used repeatedly until the equality $[V]=[W]$ is witnessed by a subvariety $Z^{\prime} \subseteq Z$ satisfying $\operatorname{dim} Z^{\prime} \leq \operatorname{dim} W$. Then we land up in the first case completing the proof.

Theorem 3.4. Let $k$ be an algebraically closed field. If Conjecture 1.3 holds for $k$, then Question 1.1 admits a positive answer over $k$.

Proof. Let $V$ and $W$ be two varieties with $[V]=[W]$ in $\mathrm{K}_{0}\left(\operatorname{Var}_{k}\right)$. Then Proposition 2.1 states that there is a variety $Z$ of dimension $t$ and $d(Z)=e$, say, disjoint from both $V$ and $W$, which witnesses this equality, i.e., $V \sqcup Z \doteqdot W \sqcup Z$. Proposition 3.3 then gives $\operatorname{dim} V=\operatorname{dim} W=: s$ and $d(V)=d(W)$.

If $s=0$, then $d(V)=d(W)$ implies $V \doteqdot W$.

If $s>0$, then we describe a procedure to reduce the sum $s+t$ in two different cases.

Case I: Suppose $s \leq t$. Let $d= \begin{cases}d(V) & \text { if } s=t, \\ 0 & \text { otherwise. }\end{cases}$

The piecewise isomorphism $V \sqcup Z \doteqdot W \sqcup Z$ gives two families $S_{1}, S_{2}, \ldots, S_{d+e}$ and $T_{1}, T_{2}, \ldots, T_{d+e}$ of irreducible subvarieties of $V \sqcup Z$ and $W \sqcup Z$ respectively of maximal dimension satisfying the hypotheses of Proposition 3.2 . Furthermore, we also get that $(V \sqcup Z) \backslash\left(\bigsqcup_{i \in[d+e]} S_{i}\right) \doteqdot(W \sqcup Z) \backslash\left(\bigsqcup_{i \in[d+e]} T_{i}\right)$.

If Conjecture 1.3 holds, we can apply Proposition 3.2 to obtain a dense subvariety $Z_{1}:=\bigsqcup_{i \in Q} T_{i}^{\prime} \subseteq Z$. The other conclusions of the proposition give the following properties:

- the variety $Z^{\prime}:=Z \backslash Z_{1}$ witnesses $[V]=[W]$, i.e., $V \sqcup Z^{\prime} \doteqdot W \sqcup Z^{\prime}$;

- $\operatorname{dim} Z^{\prime}<\operatorname{dim} Z$.

The use of Proposition 3.2 can be repeated if $\operatorname{dim} Z^{\prime} \geq s$. Hence the equality $[V]=[W]$ in $\mathrm{K}_{0}\left(\operatorname{Var}_{k}\right)$ is witnessed by some variety $Z^{\prime \prime}$ of dimension less than $s$.

Case II: Suppose $s>t$. In this case, the piecewise isomorphism $V \sqcup Z \doteqdot W \sqcup Z$ gives $V^{\prime} \subset V, W^{\prime} \subset W$ with $\operatorname{dim} V^{\prime}=\operatorname{dim} W^{\prime}<s$ such that $V \backslash V^{\prime} \doteqdot W \backslash W^{\prime}$ and $V^{\prime} \sqcup Z \doteqdot W^{\prime} \sqcup Z$.

The two cases complete the proof that $V \doteqdot W$.

\section{A presentation For $\mathrm{K}_{0}^{+}\left(\operatorname{Var}_{k}\right)$ Under Conjecture 1.3}

We assume that the Conjecture 1.3 holds (equivalently, in view of Theorem 3.4 . Question 1.1 admits a positive answer) for an algebraically closed field $k$ in this section.

For each $n \in \mathbb{Z}_{\geq 0}$ let $\operatorname{Var}_{k}^{n}$ denote the proper class of $k$-varieties of dimension at most $n$. Then $\left\{\operatorname{Var}_{k}^{n}\right\}_{n \geq 0}$ is a filtration on the objects of $\operatorname{Var}_{k}$. Further let $S_{n}$ denote the monoid, under $\sqcup$, of piecewise isomorphism classes of varieties in $\operatorname{Var}_{k}^{n}$ and $H_{n}$ denote the Grothendieck group associated with $S_{n}$ for each $n \geq 0$. If Conjecture 1.3 holds, then $H_{n}$ is the subgroup of $\mathrm{K}_{0}\left(\operatorname{Var}_{k}\right)$ generated by $S_{n}$ and thus, for each $n \in \mathbb{Z}_{\geq 0}$, the natural map $H_{n} \rightarrow H_{n+1}$ is injective.

Let $\mathfrak{M}$ denote a set of representatives of birational equivalence classes of irreducible varieties. Then $\mathfrak{M}=\bigsqcup_{n \in \mathbb{Z}_{\geq 0}} \mathfrak{M}_{n}$, where $\mathfrak{M}_{n}$ is the set of all dimension $n$ varieties in $\mathfrak{M}$. We use $\mathcal{A}, \mathcal{B}$ etc. to denote the elements of $\mathfrak{M}$. 
We say that a variety $A$ of dimension $n$ is $\mathfrak{M}$-admissible (or just admissible) if it can be embedded into some $\mathcal{A} \in \mathfrak{M}_{n}$. The assignment $A \mapsto \mathcal{A}$ is a well-defined and dimension preserving map on the class of admissible varieties. Note that every admissible variety has a unique irreducible component of maximal dimension. We say that a partition $D=\bigsqcup_{i \in[m]} D_{i}$ of a variety $D$ into locally closed subvarieties is admissible if each $D_{i}$ is admissible. Note that each variety admits an admissible partition.

Theorem 4.1. Suppose Question 1.1 admits a positive answer over an algebraically closed field $k$. Let $\mathfrak{M}$ denote a set of representatives of birational equivalence classes of irreducible $k$-varieties. Then there is a unique group isomorphism ev $_{\mathfrak{M}}$ : $\mathrm{K}_{0}^{+}\left(\operatorname{Var}_{k}\right) \rightarrow \mathbb{Z}[\mathfrak{M}]$ satisfying ev $\mathfrak{M}([\mathcal{A}])=\mathcal{A}$ for each $\mathcal{A} \in \mathfrak{M}$.

Proof. We fix $\mathfrak{M}$ and drop the subscript $\mathfrak{M}$ from $e v_{\mathfrak{M}}$. We inductively define a compatible family of maps $\left\{e v^{n}: \operatorname{Var}_{k}^{n} \rightarrow \mathbb{Z}[\mathfrak{M}]\right\}_{n \geq 0}$, where $e v^{n}$ factors through an injective group homomorphism $H_{n} \rightarrow \mathbb{Z}[\mathfrak{M}]$. By an abuse of notation, we also denote the group homomorphism by $e v^{n}$.

If $D \in \operatorname{Var}_{k}^{0}$ and $d(D)=d$, then the assignment $D \mapsto d \mathcal{U}$ clearly factors through an injective group homomorphism $H_{0} \cong \mathbb{Z} \rightarrow \mathbb{Z}[\mathfrak{M}]$, where $\mathcal{U}$ is the unique variety in $\mathfrak{M}_{0}$.

Assume by induction that $e v^{n-1}$ is a well-defined map on $\operatorname{Var}_{k}^{n-1}$ and that it factors through an injective group homomorphism $H_{n-1} \rightarrow \mathbb{Z}[\mathfrak{M}]$.

If $D \in \operatorname{Var}_{k}^{n-1}$, then define $e v^{n}(D):=e v^{n-1}(D)$ ensuring compatibility.

Let $A$ be an admissible variety of dimension $n$. Then there is an embedding $f: A \hookrightarrow \mathcal{A}$ for a unique $\mathcal{A} \in \mathfrak{M}_{n}$. Define $e v^{n}(A):=\mathcal{A}-e v^{n-1}(\mathcal{A} \backslash f(A))$.

To see that this definition does not depend on the choice of an embedding, let $g: A \hookrightarrow \mathcal{A}$ be another embedding. It will suffice to show that $e v^{n-1}(\mathcal{A} \backslash f(A))=$ $e v^{n-1}(\mathcal{A} \backslash g(A))$. Note that the following equations hold in $H_{n}$.

$$
\begin{aligned}
{[f(A)] } & =[g(A)], \\
{[f(A)]+[\mathcal{A} \backslash f(A)] } & =[g(A)]+[\mathcal{A} \backslash g(A)] .
\end{aligned}
$$

Hence $[\mathcal{A} \backslash f(A)]=[\mathcal{A} \backslash g(A)]$ in $H_{n}$. Under the hypothesis of a positive answer to Question 1.1, we conclude the same equation in $H_{n-1}$.

If $\phi: A^{\prime} \rightarrow A$ is a variety isomorphism, then $A^{\prime}$ is admissible since $A$ is and both of them embed into the same variety $\mathcal{A} \in \mathfrak{M}_{n}$. Choosing an embedding $f$ of $A$ into $\mathcal{A}$ gives an embedding $f \circ \phi$ of $A^{\prime}$ into $\mathcal{A}$. Since $f(A)=f \circ \phi\left(A^{\prime}\right)$, we have $e v^{n}(A)=e v^{n}\left(A^{\prime}\right)$.

If $A=A_{1} \sqcup A_{2}$ is a partition of an admissible variety $A$ of dimension $n$ into locally closed subvarieties and $\operatorname{dim} A_{1}=n$, then $A_{1}$ is admissible. Further if $f$ is an embedding of $A$ into $\mathcal{A}$, then using that $e v^{n-1}$ is additive we have

$$
\begin{aligned}
e v^{n}\left(A_{1}\right)+e v^{n}\left(A_{2}\right) & =\mathcal{A}-e v^{n-1}\left(\mathcal{A} \backslash f\left(A_{1}\right)\right)+e v^{n-1}\left(A_{2}\right) \\
& =\mathcal{A}-e v^{n-1}\left((\mathcal{A} \backslash f(A)) \sqcup f\left(A_{2}\right)\right)+e v^{n-1}\left(A_{2}\right) \\
& =\mathcal{A}-e v^{n-1}(\mathcal{A} \backslash f(A))-e v^{n-1}\left(f\left(A_{2}\right)\right)+e v^{n-1}\left(A_{2}\right) \\
& =\mathcal{A}-e v^{n-1}(\mathcal{A} \backslash f(A)) \\
& =e v^{n}(A) .
\end{aligned}
$$

From the previous two paragraphs it follows that whenever $A$ is admissible and $A \doteqdot B$, then $e v^{n}(A)=e v^{n}(B)$.

Now let $D=\bigsqcup_{i \in[m]} D_{i}$ be an admissible partition of a variety $D$ of dimension $n$. Define $e v^{n}(D):=\sum_{i \in[m]} e v^{n}\left(D_{i}\right)$. Any two admissible partitions of $D$ admit a common admissible refinement and, as shown above, the value of $e v^{n}\left(D_{i}\right)$ does not change under refinements. Thus $e v^{n}(D)$ is independent of the choice of an admissible partition and is well-defined. 
If $D \doteqdot D^{\prime}$, then we choose partitions $D=\bigsqcup_{i \in[m]} D_{i}$ and $D^{\prime}=\bigsqcup_{i \in[m]} D_{i}^{\prime}$ such that $D_{i}$ is isomorphic to $D_{i}^{\prime}$ for each $i$. By further refinements, we may as well assume that these partitions are admissible. Then $e v^{n}(D)=\sum_{i \in[m]} e v^{n}\left(D_{i}\right)=$ $\sum_{i \in[m]} e v^{n}\left(D_{i}^{\prime}\right)=e v^{n}\left(D^{\prime}\right)$.

This completes the proof that the map $e v^{n}$, uniquely determined by $\mathfrak{M}$, factors through an additive map $H_{n} \rightarrow \mathbb{Z}[\mathfrak{M}]$. It remains to show that $e v^{n}: H_{n} \rightarrow \mathbb{Z}[\mathfrak{M}]$ is injective.

We must show that if $D_{1}, D_{2}, D_{1}^{\prime}, D_{2}^{\prime} \in \operatorname{Var}_{k}^{n}$ satisfy $e v^{n}\left(\left[D_{1}\right]-\left[D_{2}\right]\right)=e v^{n}\left(\left[D_{1}^{\prime}\right]-\right.$ $\left.\left[D_{2}^{\prime}\right]\right)$, then $\left[D_{1}\right]-\left[D_{2}\right]=\left[D_{1}^{\prime}\right]-\left[D_{2}^{\prime}\right]$. This claim can be restated as: $e v^{n}\left(\left[D_{1}\right]+\right.$ $\left.\left[D_{2}^{\prime}\right]\right)=e v^{n}\left(\left[D_{2}\right]+\left[D_{1}^{\prime}\right]\right)$ implies $\left[D_{1}\right]+\left[D_{2}^{\prime}\right]=\left[D_{2}\right]+\left[D_{1}^{\prime}\right]$. Therefore it is sufficient to prove that if $D, D^{\prime} \in \operatorname{Var}_{k}^{n}$ satisfy $e v^{n}([D])=e v^{n}\left(\left[D^{\prime}\right]\right)$, then $[D]=\left[D^{\prime}\right]$ in $H_{n}$.

Let $D, D^{\prime} \in \operatorname{Var}_{k}^{n}$ be such that $e v^{n}([D])=e v^{n}\left(\left[D^{\prime}\right]\right)$. Looking at the " $n$ dimensional component" of this element of $\mathbb{Z}[\mathfrak{M}]$, we deduce that $\operatorname{dim} D=\operatorname{dim} D^{\prime}$ and $d(D)=d\left(D^{\prime}\right)=: d$.

Suppose $D=\bigsqcup_{i \in[t]} D_{i}$ is an admissible partition of $D$. Without loss, we may assume that $\operatorname{dim} D_{i}=n$ if and only if $i \in[d]$. For each $i \in[d]$, let $f_{i}: D_{i} \rightarrow \mathcal{A}_{i}$ be a variety embedding, where $\mathcal{A}_{i} \in \mathfrak{M}_{n}$, and set $C_{i}:=\mathcal{A}_{i} \backslash f_{i}\left(D_{i}\right)$. Then $\operatorname{dim} C_{i}<n$ and $D_{i} \sqcup C_{i} \doteqdot \mathcal{A}_{i}$. Hence $e v^{n}\left(\left[D_{i}\right]\right)+e v^{n-1}\left(\left[C_{i}\right]\right)=\mathcal{A}_{i}$ for each $i \in[d]$.

Similarly starting with an admissible partition $D^{\prime}=\bigsqcup_{i \in[s]} D_{i}^{\prime}$, where $\operatorname{dim} D_{i}^{\prime}=n$ if and only if $i \in[d]$, we obtain $\mathcal{A}_{i}^{\prime} \in \mathfrak{M}_{n}$ and $C_{i}^{\prime}$ such that $e v^{n}\left(\left[D_{i}^{\prime}\right]\right)+e v^{n-1}\left(\left[C_{i}^{\prime}\right]\right)=$ $\mathcal{A}_{i}^{\prime}$ for each $i \in[d]$. Now

$$
\begin{aligned}
\sum_{i \in[d]} \mathcal{A}_{i}+\sum_{i \in[t] \backslash[d]} e v^{n-1}\left(\left[D_{i}\right]\right) & +\sum_{i \in[d]} e v^{n-1}\left(\left[C_{i}^{\prime}\right]\right) \\
& =e v^{n}([D])+\sum_{i \in[d]} e v^{n-1}\left(\left[C_{i}\right]\right)+\sum_{i \in[d]} e v^{n-1}\left(\left[C_{i}^{\prime}\right]\right) \\
& =e v^{n}\left(\left[D^{\prime}\right]\right)+\sum_{i \in[d]} e v^{n-1}\left(\left[C_{i}^{\prime}\right]\right)+\sum_{i \in[d]} e v^{n-1}\left(\left[C_{i}\right]\right) \\
& =\sum_{i \in[d]} \mathcal{A}_{i}^{\prime}+\sum_{i \in[s] \backslash[d]} e v^{n-1}\left(\left[D_{i}^{\prime}\right]\right)+\sum_{i \in[d]} e v^{n-1}\left(\left[C_{i}\right]\right) .
\end{aligned}
$$

By comparing the components of different dimensions, we get the following equations.

$$
\begin{aligned}
\sum_{i \in[d]} \mathcal{A}_{i} & =\sum_{i \in[d]} \mathcal{A}_{i}^{\prime} \\
\sum_{i \in[t] \backslash[d]} e v^{n-1}\left(\left[D_{i}\right]\right)+\sum_{i \in[d]} e v^{n-1}\left(\left[C_{i}^{\prime}\right]\right) & =\sum_{i \in[s] \backslash[d]} e v^{n-1}\left(\left[D_{i}^{\prime}\right]\right)+\sum_{i \in[d]} e v^{n-1}\left(\left[C_{i}\right]\right) .
\end{aligned}
$$

The first equation gives that the list $\mathcal{A}_{1}, \mathcal{A}_{2}, \ldots, \mathcal{A}_{d}$ is the same as the list $\mathcal{A}_{1}^{\prime}, \mathcal{A}_{2}^{\prime}, \ldots, \mathcal{A}_{d}^{\prime}$. Since the map $e v^{n-1}$ is injective, the second equation gives $\sum_{i \in[t] \backslash[d]}\left[D_{i}\right]+$ $\sum_{i \in[d]}\left[C_{i}^{\prime}\right]=\sum_{i \in[s] \backslash[d]}\left[D_{i}^{\prime}\right]+\sum_{i \in[d]}\left[C_{i}\right]$ in $H_{n-1}$. Combining these, we obtain $[D]+\sum_{i \in[d]}\left[C_{i}\right]+\sum_{i \in[d]}\left[C_{i}^{\prime}\right]=\left[D^{\prime}\right]+\sum_{i \in[d]}\left[C_{i}^{\prime}\right]+\sum_{i \in[d]}\left[C_{i}\right]$ in $H_{n}$. Since $H_{n}$ is a group, cancelling common terms from both sides gives $[D]=\left[D^{\prime}\right]$. This completes the proof of injectivity of $e v^{n}$.

Define the map $e v: \operatorname{Var}_{k} \rightarrow \mathbb{Z}[\mathcal{M}]$ by $e v(D):=e v^{n}(D)$ whenever $D \in \operatorname{Var}_{k}^{n}$. Compatibility of the family $\left\{e v^{n}\right\}$ gives that the map $e v$ is well-defined and factors through $\mathrm{K}_{0}^{+}\left(\operatorname{Var}_{k}\right)$ to give an injective map $\mathrm{K}_{0}^{+}\left(\operatorname{Var}_{k}\right) \rightarrow \mathbb{Z}[\mathfrak{M}]$.

Since, given $C, D \in \operatorname{Var}_{k}$, there exists $n$ such that $C, D \in \operatorname{Var}_{k}^{n}$, the additivity of $e v^{n}: H_{n} \rightarrow \mathbb{Z}[\mathfrak{M}]$ for each $n$ implies that $e v: \mathrm{K}_{0}^{+}\left(\operatorname{Var}_{k}\right) \rightarrow \mathbb{Z}[\mathfrak{M}]$ is a group homomorphism. The image of $e v$ generates the group $\mathbb{Z}[\mathfrak{M}]$ since the image of $\mathfrak{M} \subseteq \operatorname{Var}_{k}$ generates the codomain. Hence $\mathrm{K}_{0}^{+}\left(\operatorname{Var}_{k}\right) \cong \mathbb{Z}[\mathfrak{M}]$. 
In characteristic 0 , Hironaka's theorem on resolution of singularities allows us to choose a set $\mathfrak{M}$ of smooth representatives of birational equivalence classes in the theorem above. Therefore it is easy to see that the above theorem subsumes both presentations for $\mathrm{K}_{0}^{+}\left(\operatorname{Var}_{k}\right)$ of Theorem 1.5 whenever $k$ is an algebraically closed field of characteristic 0 .

\section{The Associated graded RING OF $\mathrm{K}_{0}\left(\operatorname{Var}_{k}\right)$}

We continue to work under the hypothesis of a positive answer to question 1.1 over an algebraically closed field of characteristic 0 in this section. Under this hypothesis, the usual dimension function factorizes through the the Grothendieck group.

Two varieties $X$ and $Y$ of dimension $n$ are birational if and only if there are open subvarieties $X^{\prime} \subseteq X$ and $Y^{\prime} \subseteq Y$ such that $X^{\prime} \cong Y^{\prime}$ if and only if $\operatorname{dim}([X]-[Y])=$ $\operatorname{dim}\left(\left[X \backslash X^{\prime}\right]-\left[Y \backslash Y^{\prime}\right]\right)<n$, where $[X]$ denotes the class of the variety $X$ in $\mathrm{K}_{0}\left(\operatorname{Var}_{k}\right)$.

In general the product of two varieties in $\mathfrak{M}$ is birational (but not necessarily equal) to a variety in $\mathfrak{M}$. This suggests looking at the structure of the associated graded ring of $\mathrm{K}_{0}\left(\operatorname{Var}_{k}\right)$, where the grading on $\mathrm{K}_{0}\left(\operatorname{Var}_{k}\right)$ is induced by dimension. Let $\left\{F_{n}\right\}_{n \geq 0}$ be the filtration on $\mathrm{K}_{0}\left(\operatorname{Var}_{k}\right)$ induced by dimensions and let $\mathfrak{G}$ denote the associated graded ring of $\mathrm{K}_{0}\left(\operatorname{Var}_{k}\right)$ with respect to this filtration.

The construction of $\mathfrak{G}$ is as follows. Set $F_{-1}:=\{0\}$ for technical purposes. Let $G_{n}:=F_{n} / F_{n-1}$ for each $n \geq 0$ and let $\mathfrak{G}$ denote the abelian group $\bigoplus_{n \geq 0} G_{n}$. There are multiplication maps $G_{n} \times G_{m} \rightarrow G_{n+m}$ defined by $\left(x+F_{n-1}\right)\left(y+F_{m-1}\right)=$ $x y+F_{n+m-1}$ for each $n, m \geq 0$. These maps combine to give a multiplication structure on $\mathfrak{G}$.

Let $\mathfrak{B}_{n}$ denote the set of birational equivalence classes of irreducible varieties of dimension $n$ and let $\mathfrak{B}:=\bigsqcup_{n>0} \mathfrak{B}_{n}$. The set $\mathfrak{B}$ carries a monoid structure induced by the multiplication of varieties, where the class of a singleton acts as the identity. The usual dimension function on varieties factors through $\mathfrak{B}$.

Theorem 5.1. Suppose Question 1.1 admits a positive answer over an algebraically closed field $k$. The associated graded ring $\mathfrak{G}$ of $\mathrm{K}_{0}\left(\operatorname{Var}_{k}\right)$ with respect to the dimension grading is the monoid ring $\mathbb{Z}[\mathfrak{B}]$, where $\mathfrak{B}$ is the multiplicative monoid of birational equivalence classes of irreducible varieties.

Proof. Since Question 1.1 admits a positive answer over $k$, we can use the group isomorphism of Theorem 4.1 induced by the evaluation map to define a multiplicative structure on $\mathbb{Z}[\mathfrak{M}]$. By an abuse of notation, we will say that $\left\{F_{n}\right\}_{n \geq 0}$ is a filtration on $\mathbb{Z}[\mathfrak{M}]$ and $\mathfrak{G}$ is its associated graded ring.

Let $\mathcal{A} \mapsto[[\mathcal{A}]]$ denote the canonical bijection $\mathfrak{M} \rightarrow \mathfrak{B}$, which takes an irreducible variety to its birational equivalence class. This clearly extends to a group isomorphism $\Phi: \mathfrak{G} \rightarrow \mathbb{Z}[\mathfrak{B}]$. We show that $\Phi$ also preserves multiplication.

Given $\mathcal{A} \in \mathfrak{M}_{n}$ and $\mathcal{B} \in \mathfrak{M}_{m}$, the product $\mathcal{A} \times_{\text {Spec } k} \mathcal{B}$ is irreducible and thus is birational to a unique $\mathcal{C} \in \mathfrak{M}_{n+m}$. In other words, $\left(\mathcal{A}+F_{n-1}\right) \cdot\left(\mathcal{B}+F_{m-1}\right)=$ $\mathcal{C}+F_{n+m-1}$ in $\mathfrak{G}$. We also have $[[\mathcal{C}]]=\left[\left[\mathcal{A} \times{ }_{\text {Spec } k} \mathcal{B}\right]\right]=[[\mathcal{A}]][[\mathcal{B}]]$ in the monoid $\mathfrak{B}$. Hence $\Phi\left(\left(\mathcal{A}+F_{n-1}\right) \cdot\left(\mathcal{B}+F_{m-1}\right)\right)=\Phi\left(\mathcal{A}+F_{n-1}\right) \cdot \Phi\left(\mathcal{B}+F_{m-1}\right)$. This shows that $\Phi$ preserves multiplication on the image of $\mathfrak{M}$ in $\mathfrak{G}$. It is routine to verify that $\Phi$ is multiplicative on the whole of $\mathfrak{G}$. 


\section{Further REMARKS}

Recall that an element $a$ of a ring $R$ is said to be regular if it is not a zero divisor in $R$. The following question is important for better understanding of the Grothendieck ring and is open even in the case of algebraically closed fields.

Question 6.1. Let $k$ be a field of characteristic 0 . Is $\mathbb{L}:=\left[\mathbb{A}_{k}^{1}\right]$ a regular element of $\mathrm{K}_{0}\left(\operatorname{Var}_{k}\right)$ ?

Lemma 4.8 and Proposition 4.9 in [10] connect this question to Question 1.1] in special cases, but no further development has been made.

The model-theoretic Grothendieck ring $\mathrm{K}_{0}(k)$ of an algebraically closed field $k$, as defined in [4], is a quotient of $\mathrm{K}_{0}\left(\operatorname{Var}_{k}\right)$. It is natural to ask the following question.

Question 6.2. Is the model-theoretic Grothendieck ring $\mathrm{K}_{0}(k)$ isomorphic to $\mathrm{K}_{0}\left(\operatorname{Var}_{k}\right)$ for an algebraically closed field $k$ ?

Acknowledgements. I would like to thank Prof. Mike Prest for discussions and for careful reading of the paper. I would also like to thank Profs. Thomas Scanlon, François Loeser and Julien Sebag for useful comments on the first draft of the paper. I am grateful to Adam Biggs and Andrew Davies for discussions on Grothendieck rings and algebraic geometry.

\section{REFERENCES}

[1] F. Bittner, The universal Euler characteristic for varieties of characteristic zero, Compos. Math., 140(4) (2004), 1011-1032.

[2] M. Gromov, Endomorphisms of symbolic algebraic varieties, J. Eur. Math. Soc., 1(2) (1999), 109-197.

[3] J. Kollár, Conics in the Grothendieck ring, Adv. Math., 198(1) (2005), 27-35.

[4] J. Krajicěk and T. Scanlon, Combinatorics with definable sets: Euler characteristics and Grothendieck rings, Bull. Symb. Logic, 6(3) (2000), 311-330.

[5] S. Lamy and J. Sebag, Birational self-maps and piecewise algebraic geometry, J. Math. Sci. -Univ. Tokyo, 19(3) (2012), 325-357.

[6] M. Larsen and V. A. Lunts, Motivic measures and stable birational geometry, Mosc. Math. J., 3(1) (2003), 85-95.

[7] Q. Liu and J. Sebag, The Grothendieck ring of varieties and piecewise isomorphisms, Math. Z., 265(2) (2010), 321-342.

[8] B. Poonen, The Grothendieck ring of varieties is not a domain, Math. Res. Lett., 9(4) (2002), 493-497.

[9] N. Sahasrabudhe, Grothendieck ring of varieties, Master thesis at Université de Bordeaux, available at http://www.algant.eu/documents/theses/neeraja.pdf.74, 2007.

[10] J. Sebag, Variations on a question of Larsen and Lunts, Proc. Amer. Math. Soc., 138(4) (2010), 1231-1242. 